\title{
Structural and Operating Optimization of the Methanol Process Using a Metaheuristic Technique
}

\section{Supplementary Material}

\author{
Luis German Hernandez-Perez, ${ }^{a}$ Abdulrahman $S$. \\ Alsuhaibani, ${ }^{b}$ Neyara Radwan, ${ }^{c, d}$ Mahmoud M. El-Halwagi, ${ }^{b}$ \\ Jose Maria Ponce-Ortega ${ }^{a^{*}}$
}

${ }^{a}$ Chemical Engineering Department, Universidad Michoacana de San Nicolás de Hidalgo, Morelia, Mich., 58060, México

${ }^{b}$ Chemical Engineering Department, Texas A\&M University, College Station TX, 77843, USA

${ }^{c}$ King Abdulaziz University, Box 80204, Jeddah, Saudi Arabia

${ }^{d}$ Mechanical Department, Faculty of Engineering, Suez Canal University, Ismalia, 41522, Egypt.

*Author for Correspondence:

Prof. José María Ponce-Ortega

Universidad Michoacana de San Nicolás de Hidalgo

Chemical Engineering Department

Tel: +52 (443) 3273584; Fax: +52 (443) 3273584

E-mail: jmponce@umich.mx 


\section{Content (11 pages, 9 figures):}

This supporting information (11 pages) presents Figures S1 to S9 where the results obtained to different cases using different termination criteria, which are additional results to the ones presented in the paper.

Figure S1: Results at SSTC for Case 1a reached at Generation 68.

Figure S2: Results at SSTC for Case 1b reached at Generation 68.

Figure S3: Results at SSTC for Case 2a reached at Generation 52.

Figure S4: Results at SSTC for Case $2 b$ reached at Generation 95.

Figure S5: Results at ChiTC for Case 3 reached at Generation 43.

Figure S6: Results at ChiTC for Case 4 reached at Generation 83.

Figure S7: Results at SSTC for Case 5 reached at Generation 72.

Figure S8: Results at SSTC for Case 6 reached at Generation 78.

Figure S9: Results after MNG for Case 7 reached at Generation 100. 


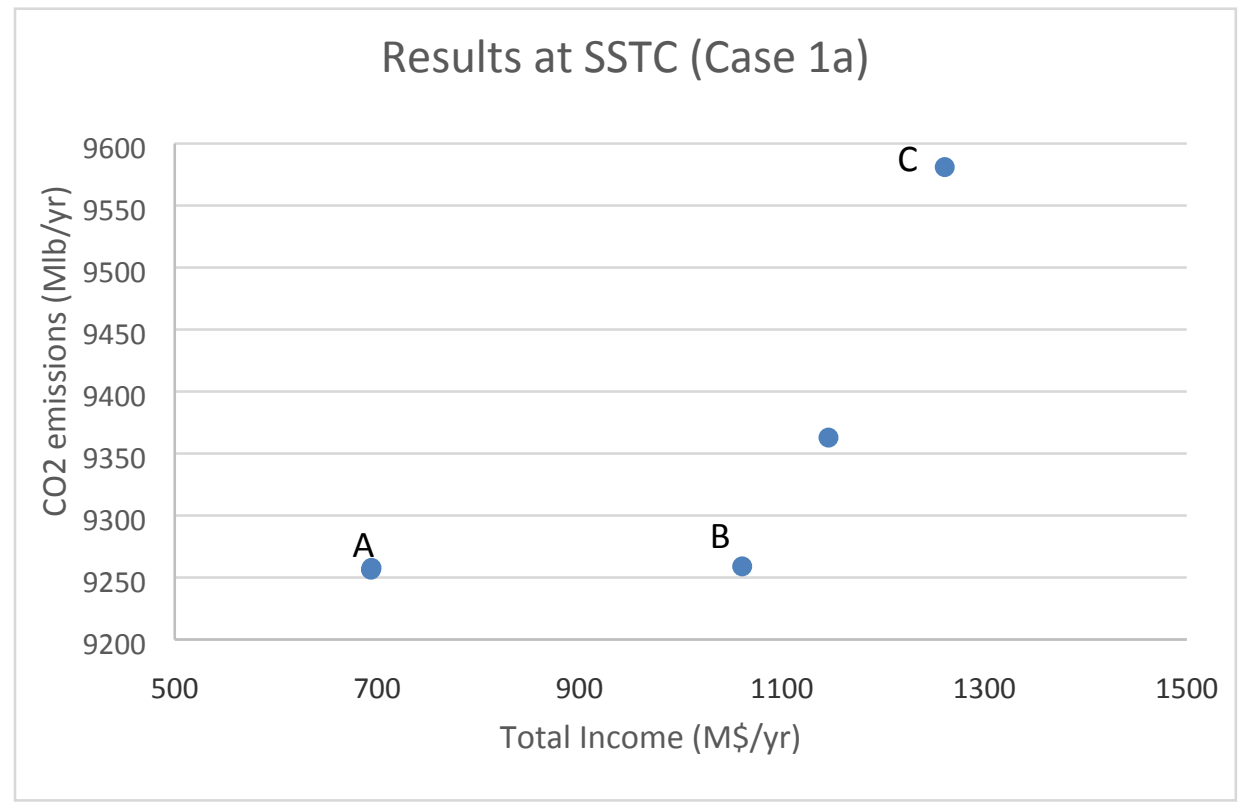

Figure S1. Results at SSTC for Case 1a reached at Generation 68. 


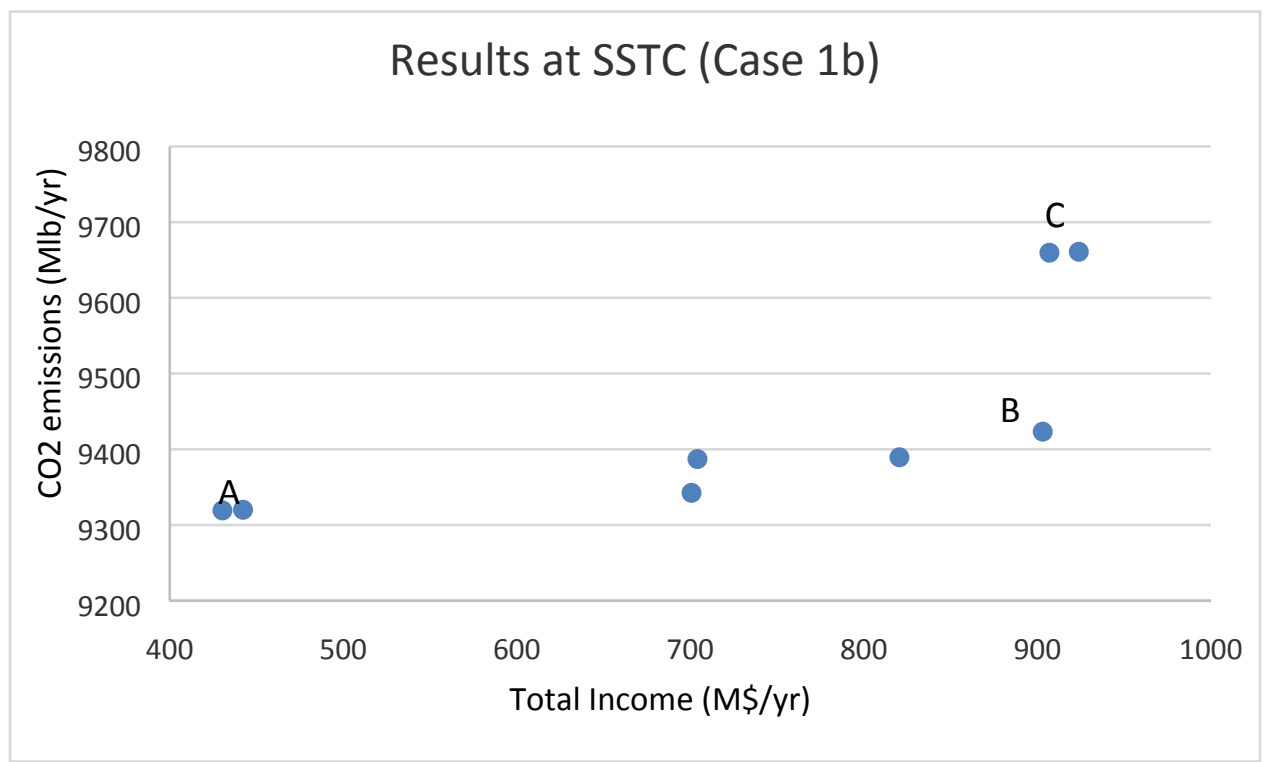

Figure S2. Results at SSTC for Case $1 \mathrm{~b}$ reached at Generation 68. 


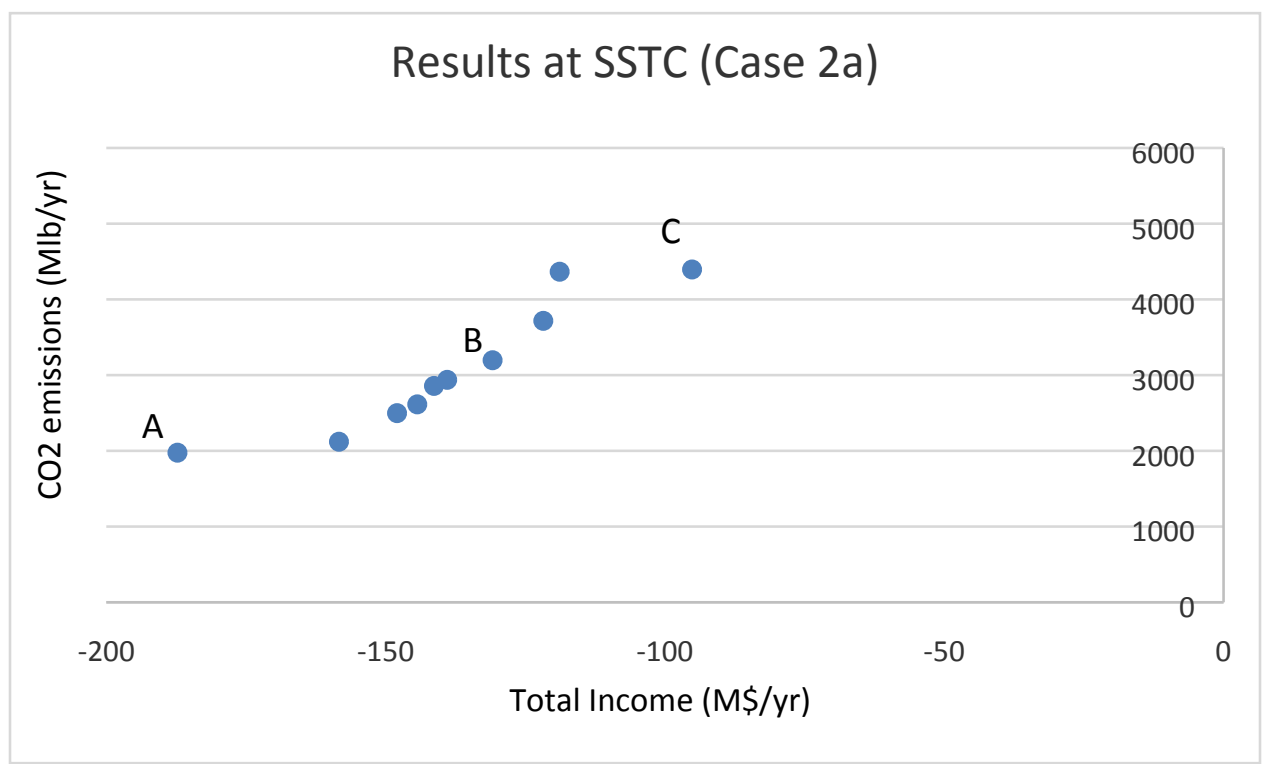

Figure S3. Results at SSTC for Case 2a reached at Generation 52. 


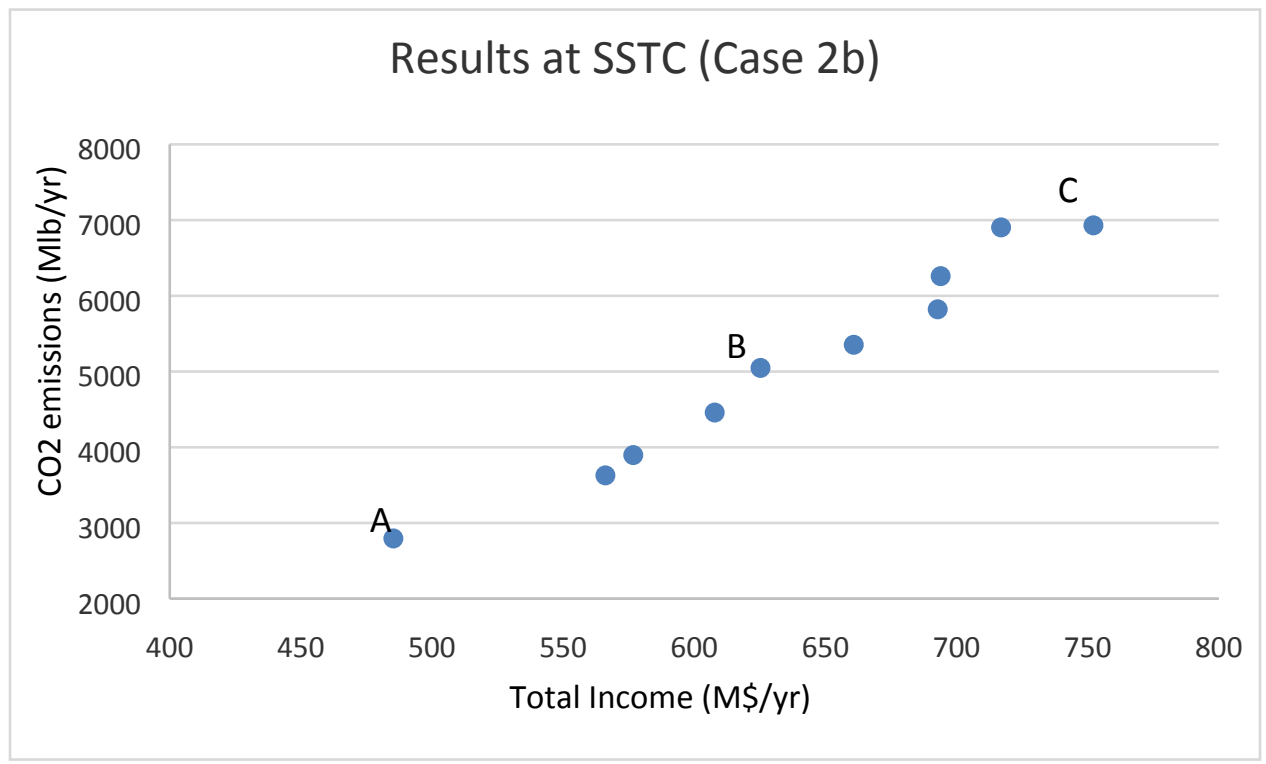

Figure S4. Results at SSTC for Case 2b reached at Generation 95. 


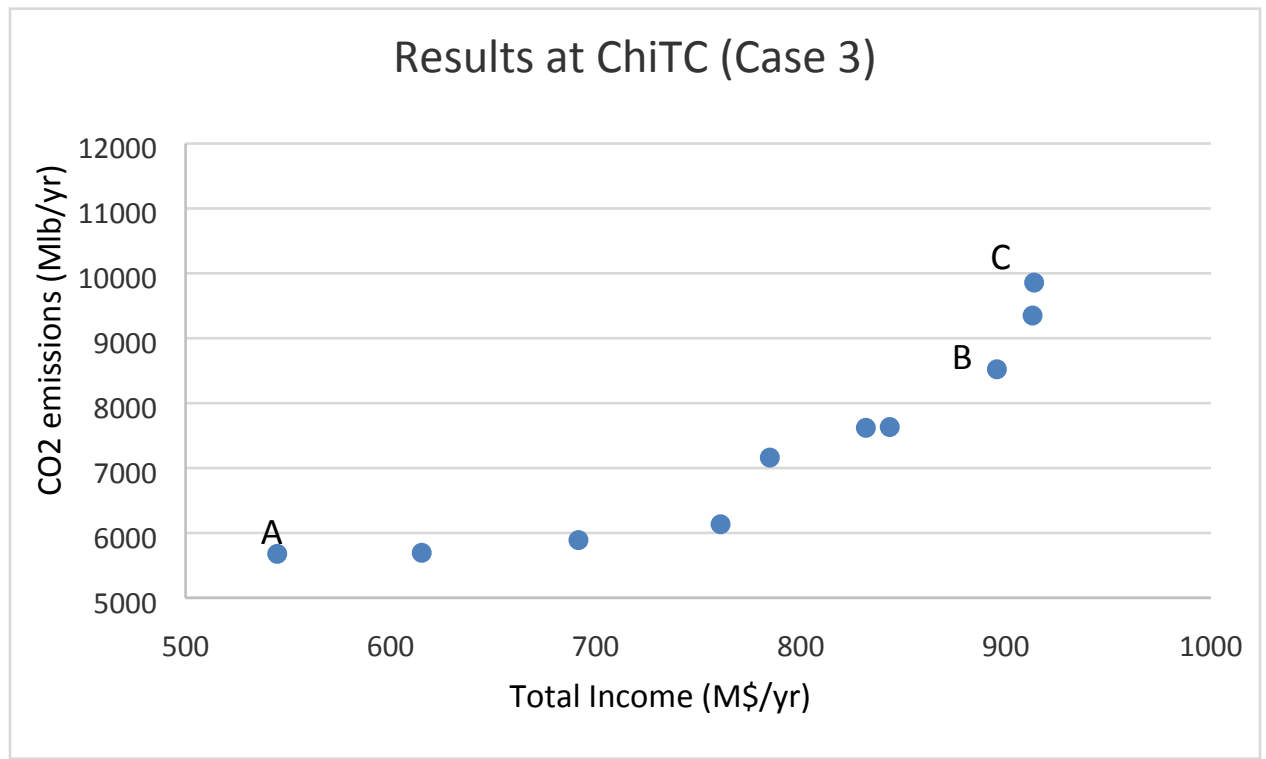

Figure S5. Results at ChiTC for Case 3 reached at Generation 43. 


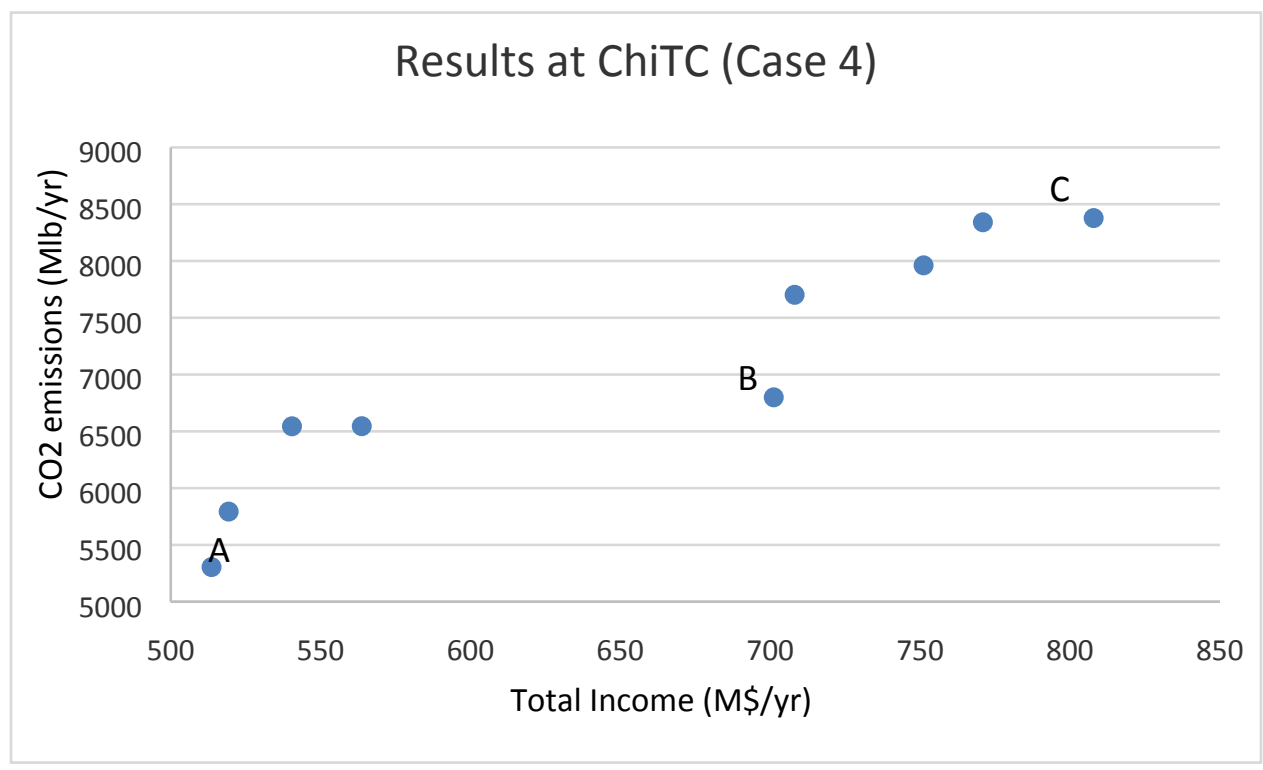

Figure S6. Results at ChiTC for Case 4 reached at Generation 83. 


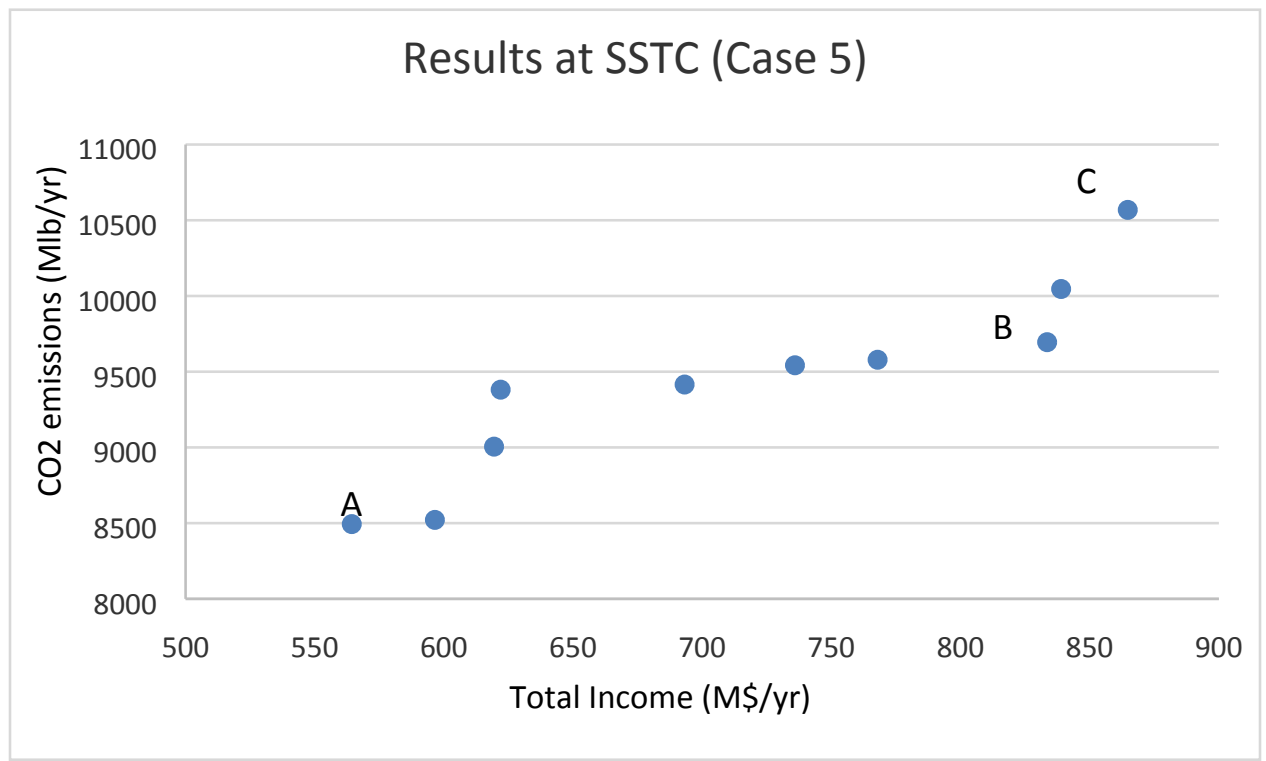

Figure S7. Results at SSTC for Case 5 reached at Generation 72. 


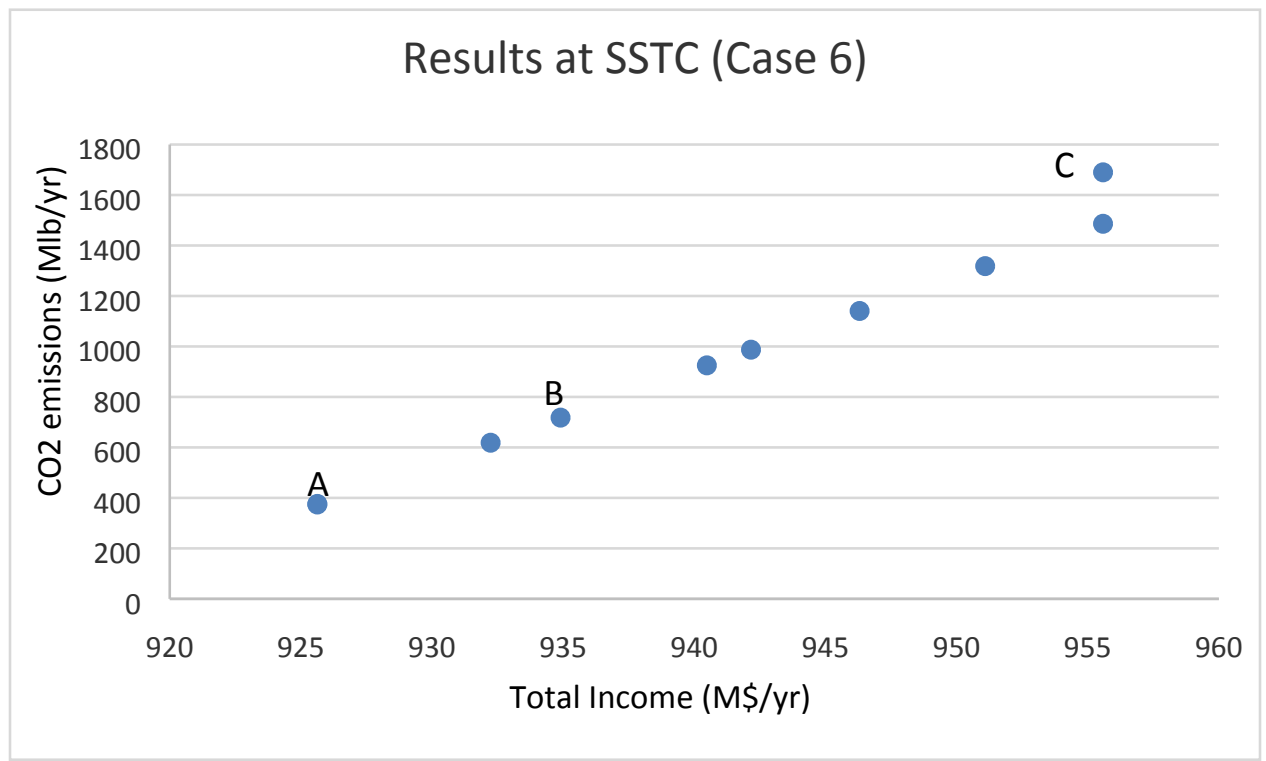

Figure S8. Results at SSTC for Case 6 reached at Generation 78. 


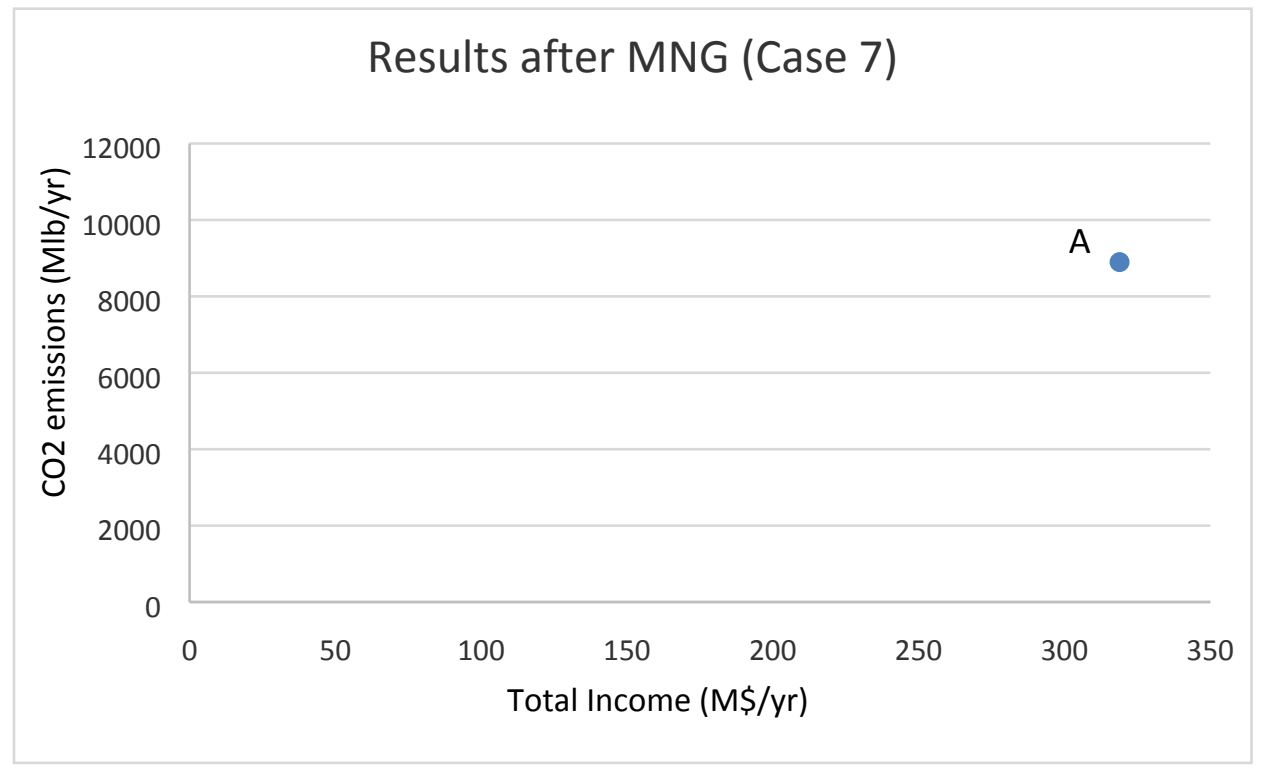

Figure S9. Results after MNG for Case 7 reached at Generation 100. 